\title{
Gastrointestinale Komplikationen bei hämatologischen Patienten
}

Patienten mit hämatologischen Erkrankungen (z.B. mit akuten und chronischen myeloischen und lymphatischen Leukämien, myelodysplastischem Syndrom, multiplem Myelom, Hodgkin oder Non-Hodgkin-Lymphomen) sind aufgrund krankheitsassoziierter und therapiebedingter Risiken einer Vielzahl an Komplikationen ausgeliefert. Neben neurologischen, pulmonalen und hepatosplenischen spielen insbesondere gastrointestinalen(GI) Komplikationen mit einer hohen Morbidität und Mortalität eine große Rolle [Coy DL et al. RadioGraphics 2005; 25: 305-318]. Um eine angemessene und schnelle Therapie einleiten zu können, sind die Kliniker häufig auf die Expertise des Radiologen angewiesen. Die unten aufgeführte Tabelle gibt eine Übersicht über gastrointestinale Komplikationen bei hämatologischen Patienten und schlägt eine Einteilung in nicht infektiöse und infektiöse Komplikationen vor ( Tab.1).

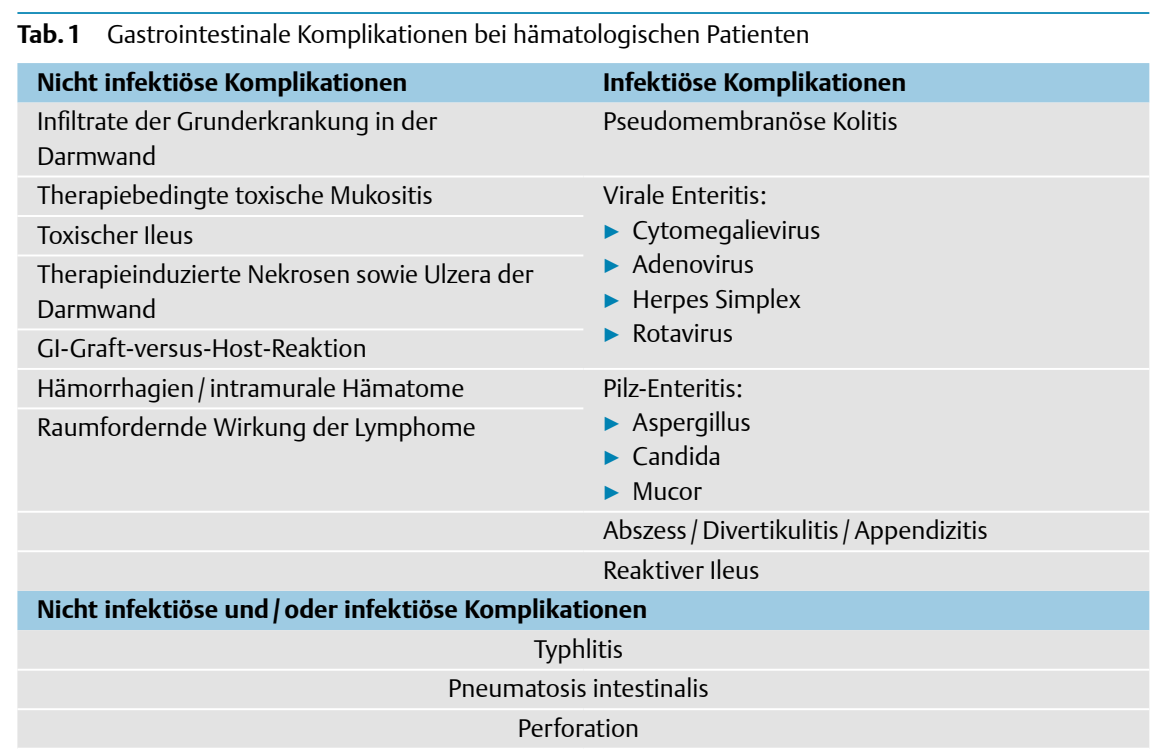




\section{Nicht infektiöse Komplikationen} $\nabla$

Direkte gastrointestinale

Wirkungen von hämatologischen Erkrankungen

Direkte gastrointestinale Wirkungen der hämatologischen Erkrankungen beruhen meistens auf einer Tumorzellinfiltration der Darmwand oder dem raumfordernden Aspekt mesenterialer und retroperitonealer Lymphome. Die Darmkompression und -konstriktion kann zu Passageverzögerung, Intussuszeption und mechanischem Ileus führen. Schleimhautulzerationen

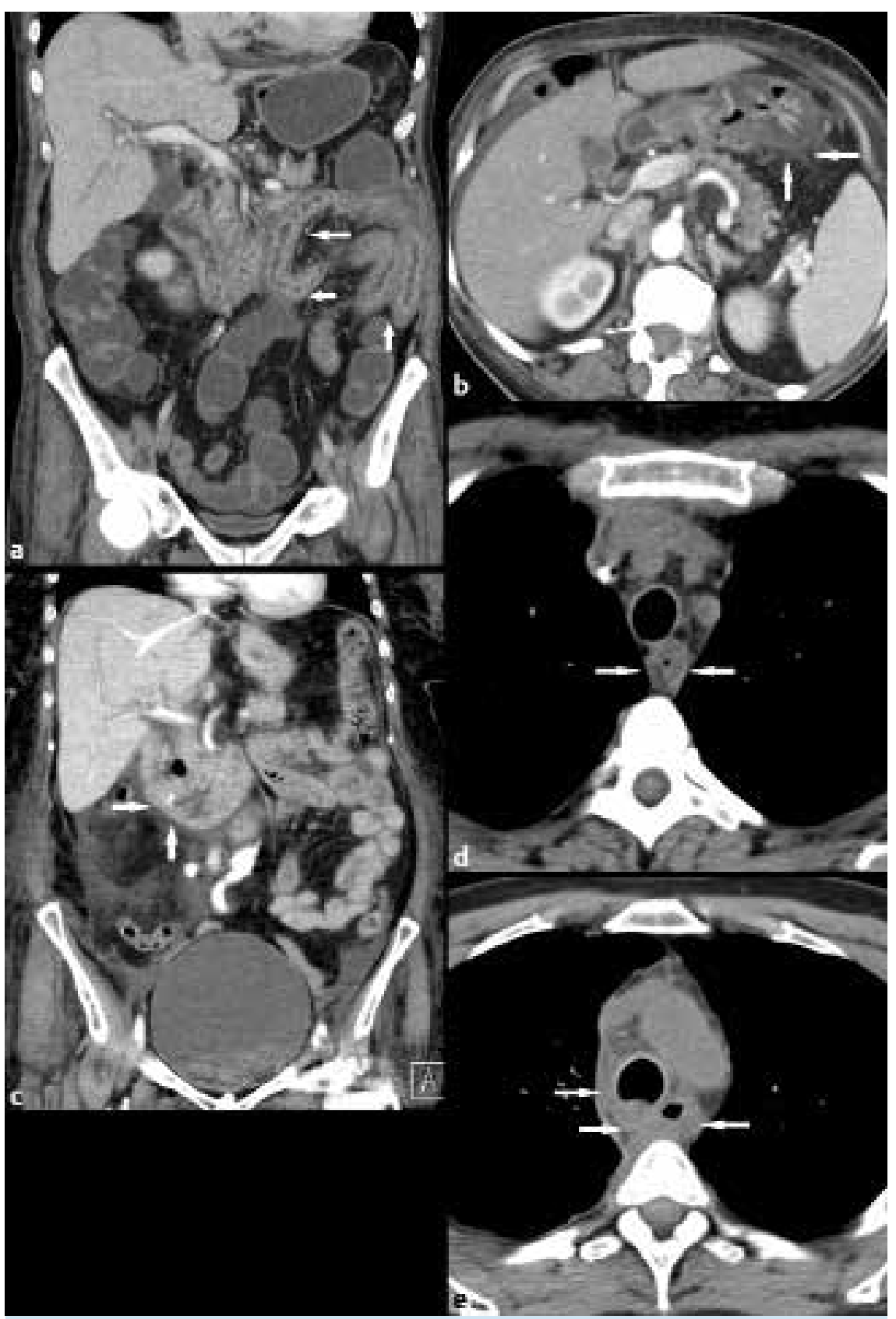

Abb. 1 Bildbeispiele von Mukositis unter Konditionierungstherapie bei Patienten mit hämatologischer maligner Erkrankung. a Der Dünndarm ist typischerweise langstreckig befallen mit deutlicher Wandverdickung, submukösem Ödem, insgesamt Darmwandinduration (Pfeile) und Erweiterung der mesenterialen Gefäße. Die Umgebungsreaktion (Stranding, Aszites) ist dabei typischerweise sehr diskret. $\mathbf{b}$ demonstriert eine Magen betonte (Pfeile) Mukositis. Eine isolierte Mukositis im Duodenum (Pfeile) ist selten (c), meistens tritt sie als Teil einer Panenteritis auf. Der Befall des Ösophagus ist sehr häufig und macht sich bei den Betroffenen früh aufgrund von schweren Schluckstörungen bemerkbar. In der Bildgebung wird es leider in den sonst meist nativ durchgeführten Thorax-CTs vernachlässigt (d). Typisch ist eine konzentrische Wandverdickung (Pfeile), zum Teil mit irregulärer Wandberandung nach innen und oft intraluminalen Blutkoagelen oder „food trapping“. Selten kann die Entzündung auf das benachbarte Mediastinum (Pfeile) übergreifen (e). Differenzialdiagnostisch muss hier an eine Candidose („Soor“) gedacht werden.

können gastrointestinale Blutungen verursachen.

\section{Therapiebedingte toxische}

\section{Mukositis}

Die Mukositis ist eine unerwünschte, jedoch unvermeidbare Nebenwirkung intensiver Chemo- und Strahlentherapien bzw. der Konditionierungstherapie zur Stammzelltransplantation hämatologischer Erkrankungen und kommt durch toxisch bedingte Veränderungen der Schleimhautbarriere zustande. Sie kann den gesamten Verdauungstrakt betreffen und läuft in 4 Phasen ab:

1. In der inflammatorischen Phase steht die Induktion der proinflammatorischen Zytokine Interleukin-1, Tumornekrosefaktor- $\alpha$ und Interferon- $\gamma$ durch die zytotoxischen Medikamente und die Bestrahlung im Vordergrund. Diese Zytokine induzie-

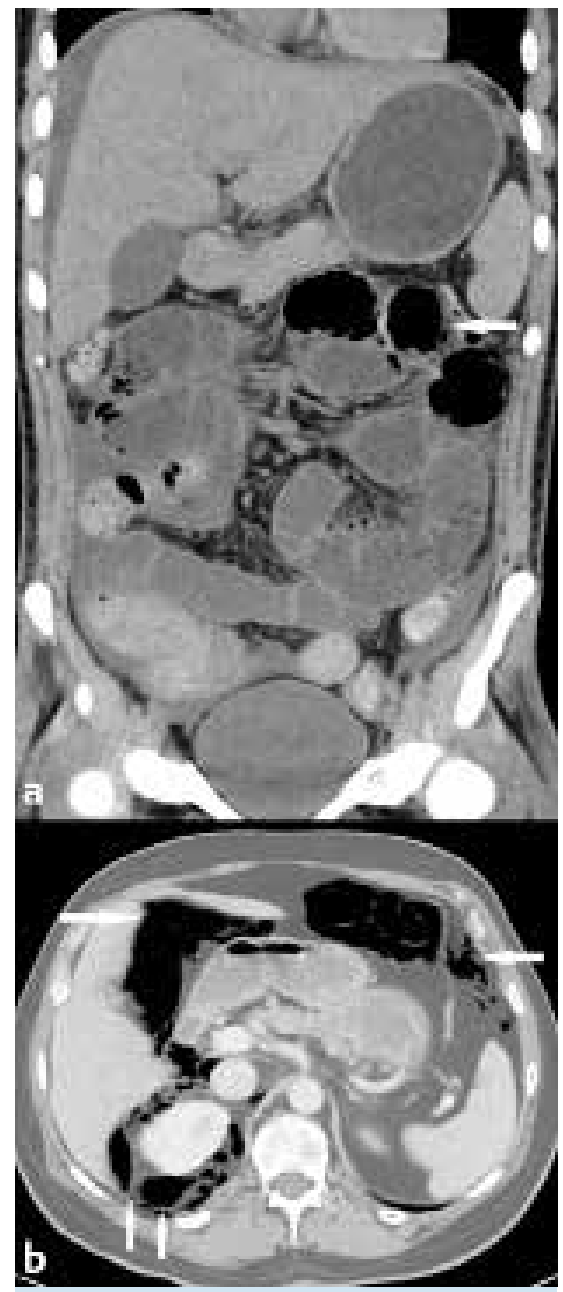

Abb. 2 Eine Darmperforation kann sowohl nach intraperitoneal (Pneumoperitoneum) (a) als auch nach retroperitoneal stattfinden (b, Pfeile), z. B. infolge von Schleimhautulzerationen. 


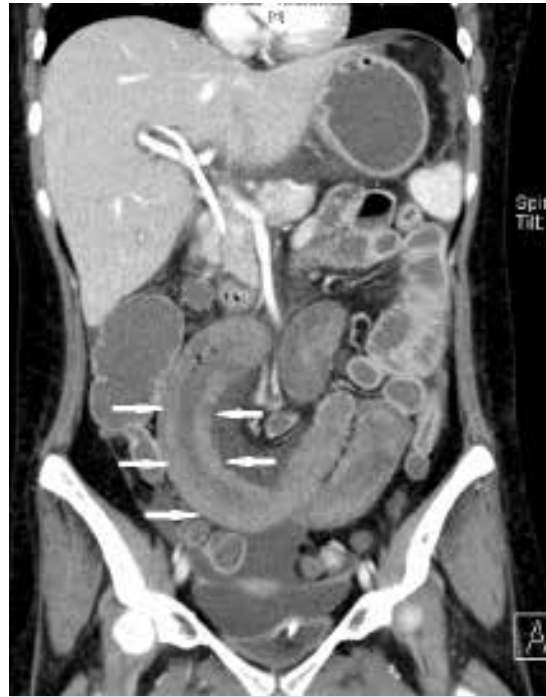

Abb. 3 In der Phase der Thrombopenie, aber auch infolge von Mukositis (bowel mucosal injury) kann es zur Gl-Blutungen mit intraluminalen Hämatomen (Pfeile) oder intramuralen Hämatomen kommen.

ren Veränderungen der Schleimhautfunktion, -permeabilität, -integrität und des -metabolismus, wobei die Epithelzellen noch intakt bleiben;

2. In der epithelialen Phase kommt es zu einer Schleimhautatrophie, -verdünnung und -nekrose, da die schnell proliferierenden Schleimhautzellen in ihrer Erneuerung gehemmt werden;

3. Dies führt innerhalb von 14 Tagen nach Einleitung der Chemotherapie zur ulzerativen Phase;

4. Die letzte Phase stellt die Heilungsphase dar.
Die Mukositis präsentiert sich klinisch mit moderaten bis schweren GI-Symptomen wie sekretorischer bis blutiger Diarrhoe, generalisierten abdominellen Schmerzen, Darmatonie, Übelkeit und Erbrechen. Typische computertomografische Befunde bei der Mukositis sind Darmwandverdickung, Schleimhauthyperämie oder sogar eine Mosaikperfusion. Diese können segmental oder diffus im gesamten Verdauungstrakt auftreten. Zusätzliche perienterale Fettgewebsimbibierung, Aszites und Lymphknotenvergrößerungen sind meistens im Gegensatz zu infektiösen Darmkomplikationen gering ausgeprägt ( $\bullet$ Abb. 1 ) [Schmit M et al. AJR Am J Roentgenol 2008; 190: 712-719].

\section{Weitere therapiebedingte GI-Komplikationen}

Durch die schnelle Lymphomrückbildung unter Chemotherapie können Lücken in der Darmwand und nicht gerade selten Darmperforationen entstehen, welche sich in der Bildgebung als freie oder gedeckte intra- oder retroperitoneale Luftansammlungen sichtbar machen (○ Abb.2). Der gleiche Mechanismus kann zu Gefäßschäden in der Darmwand führen, deren Folge eine Hämorrhagie nach intraluminal oder intramural oder intraperitoneale Hämatome sein können ( $\triangle$ Abb. 3) [Schmit M et al. AJR Am J Roentgenol 2008; 190: 712-719; Keats TE et al. AJR Am J Roentgenol 1974; 122: 150-152; Day DL et al. AJR Am J Roentgenol 1988; 151: 85-87; Bates FT et al. AJR Am J Roentgenol 1989; 152: 991-994].

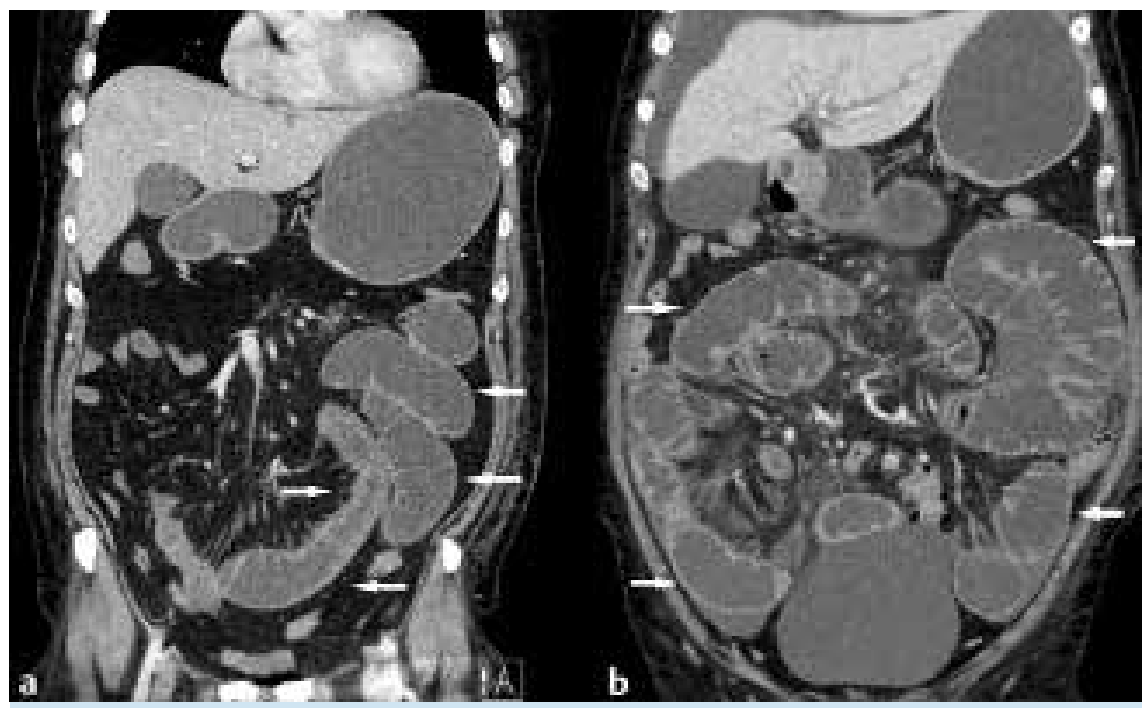

Abb. 4 Infolge der Medikamententoxizität kommt es zu paralytischen Ileuszuständen (Pfeile), die unbedingt von mechanischen Passagestörungen unterschieden werden sollten. Die Umgebungsreaktion in solchen Fällen bleibt lange dezent.
Der toxische Ileus ist eine bekannte Komplikation/Nebenwirkung vieler Chemotherapeutika. Wichtig ist hier die Differenzierung von einem mechanischen Ileus insbesondere bei Patienten in der Neutropenie, da durch orale Nahrungskarenz und Einlage einer Entlastungssonde sich die meisten Fälle rasch bessern (० Abb. 4).

\section{Graft-versus-Host-Reaktion}

Die Graft-versus-Host-Krankheit entsteht durch die Reaktion von immunkompetenten Spender-T-Zellen gegen Ober-

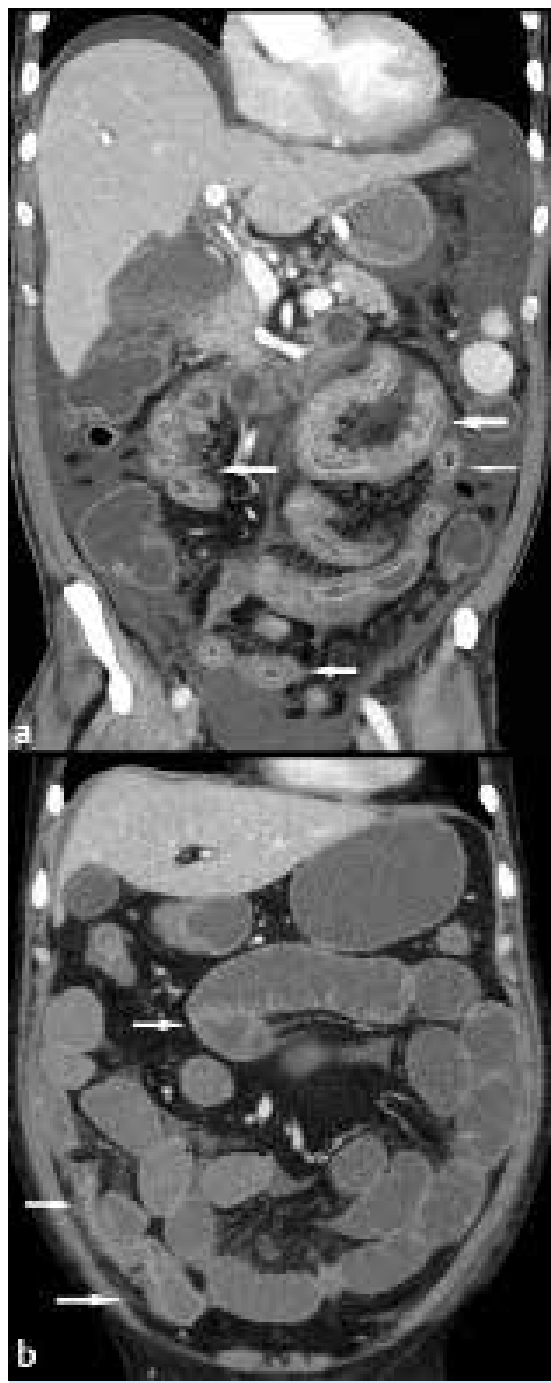

Abb. 5 GI-GVHD-Fälle in unterschiedlichen Phasen dieser Komplikation. In der subakuten Phase lässt sich eine meist diffuse, hauptsächlich das lleum betreffende Darmwandverdickung mit dicker, KM-aufnehmender Schleimhaut (Granulationsgewebe) (Pfeile) nachweisen (a). Im weiteren Verlauf hingegen normalisiert sich der Darmwandbefund mit nur leichter Persistenz vormaliger Veränderungen am terminalem lleum und oft auch am Zökum (b). 


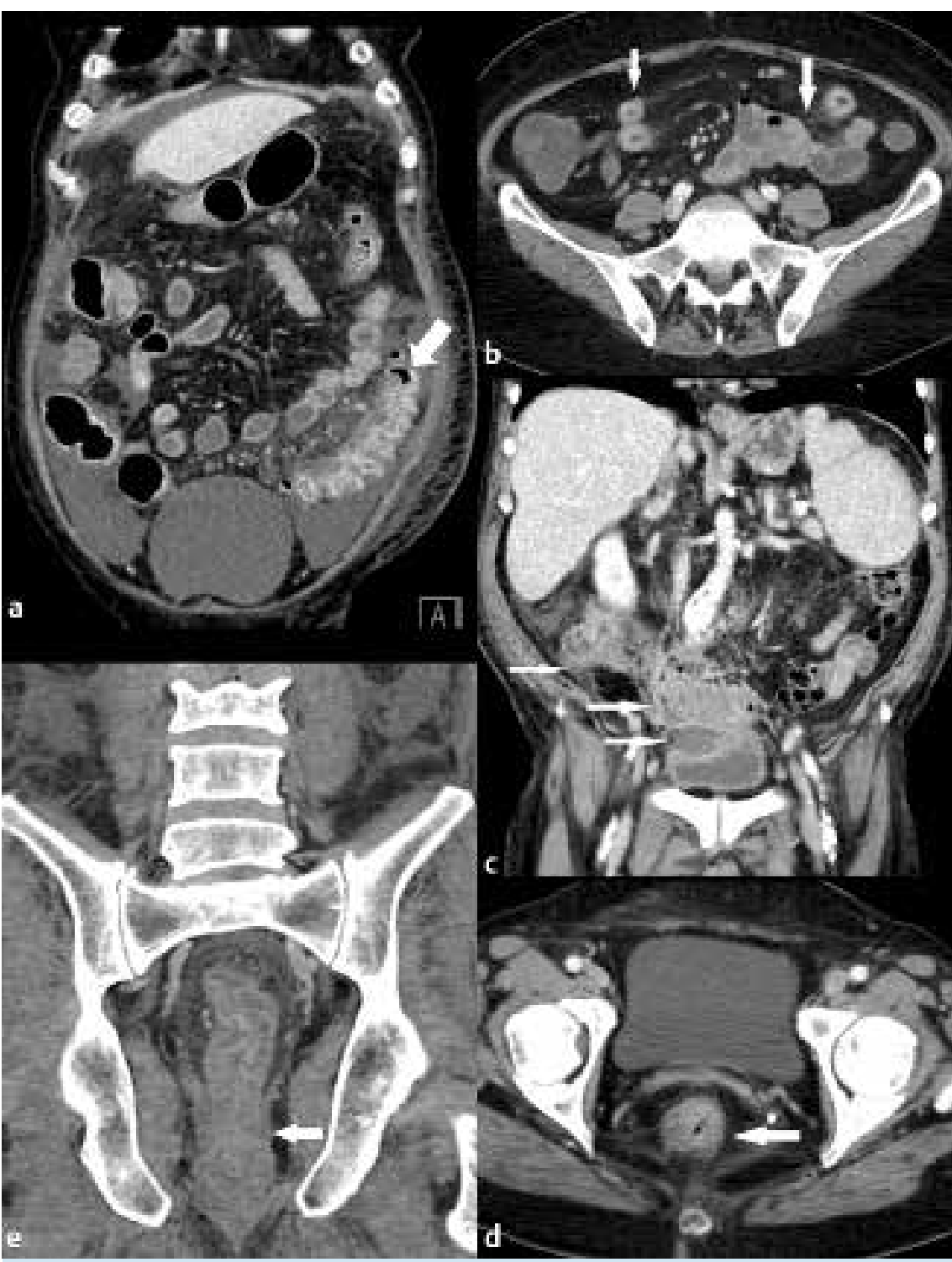

Abb. 6 Beispiele von infektiösen bakteriellen GI-Komplikationen (Pfeile): Pseudomembranöse Colitis (a), Enteritis (b), Enterocolitis mit Abszedierung (c) sowie Proctitis (d-e).

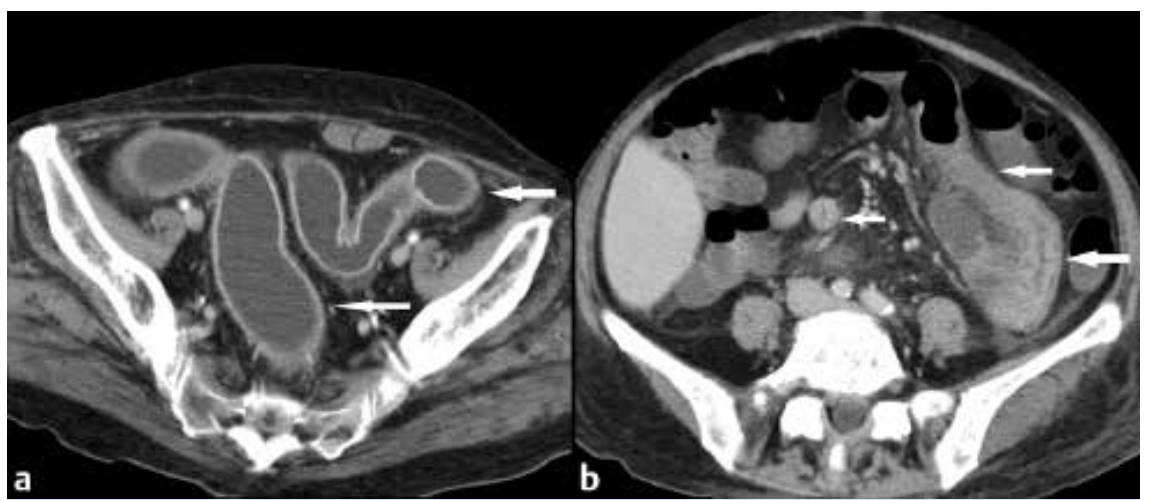

Abb. 7 Bildbeispiele von viralen GI-Komplikationen: CMV-Colitis (a, Pfeil) und Rotavirus-Enteritis (b, Pfeil). flächenantigene auf Empfängergewebe. Dadurch wird eine zelluläre und humorale Immunkaskade ausgelöst, bei der spezifische, gegen den Wirt gerichtete zytotoxische T-Zellen und Antikörper gebildet werden. Die akute Graft-versus-HostReaktion tritt innerhalb der ersten Wochen nach Transplantation auf und betrifft vor allem die Haut, die Leber und den Verdauungstrakt. Bei einer Beteiligung des Verdauungstrakts sind die vorherrschenden Symptome Diarrhoen, Bauchschmerzen und Tenesmen. Dank der prophylaktischen immunsuppressiven Therapie mit Antithymozytenglobulin, Ciclosporin A, evtl. in Kombination mit Methotrexat oder Kortikosteroiden und Calcineurinhemmer, sind die Inzidenz der akuten Graft-versus-Host-Reaktion sowie die Mortalität gesunken [Schmit M et al. AJR Am J Roentgenol 2008; 190: 712-719]. Der typische CTBefund der gastrointestinalen akuten Graft-versus-Host-Reaktion ist das erhöhte Enhancement der Schleimhaut des gesamten Verdauungstrakts. Histopathologisch lässt sich dieser Befund durch das hoch vaskularisierte Granulationsgewebe erklären, das die zerstörte Mukosa ersetzt. Falls eine Darmwandverdickung vorliegt, betrifft sie zumeist den Dünndarm ( $\bullet$ Abb. 5a). Weitere häufige CTBefunde sind flüssigkeitsgefüllte und dilatierte Darmschlingen, verdickte Darmfalten (comb sign), submuköses Ödem und Dissoziation der Darmschlingen. Seltener findet sich eine Imbibierung des perienteralen Fettgewebes ( $\bullet$ Abb. $\mathbf{5} \mathbf{b}$ ). Typische extraintestinale abdominale CT-Befunde sind Hepatomegalie, Aszites sowie ein erhöhtes Enhancement der Gallenblasen- und Harnblasenwand [Schmit M et al. AJR Am J Roentgenol 2008; 190: 712-719; Donnelly LF. AJR Am J Roentgenol 1996; 167: 909-913; Coy DL et al. RadioGraphics 2005; 25: 305-318]. Die chronische Graft-versus-Host-Reaktion des Verdauungstrakts tritt in der späten Posttransplantationphase auf, welche 100 Tage nach der Transplantation beginnt. Vorherrschendes Symptom ist die Malabsorption. Die Bildgebung zeigt meistens keine oder nur diskrete Abnormalitäten. 


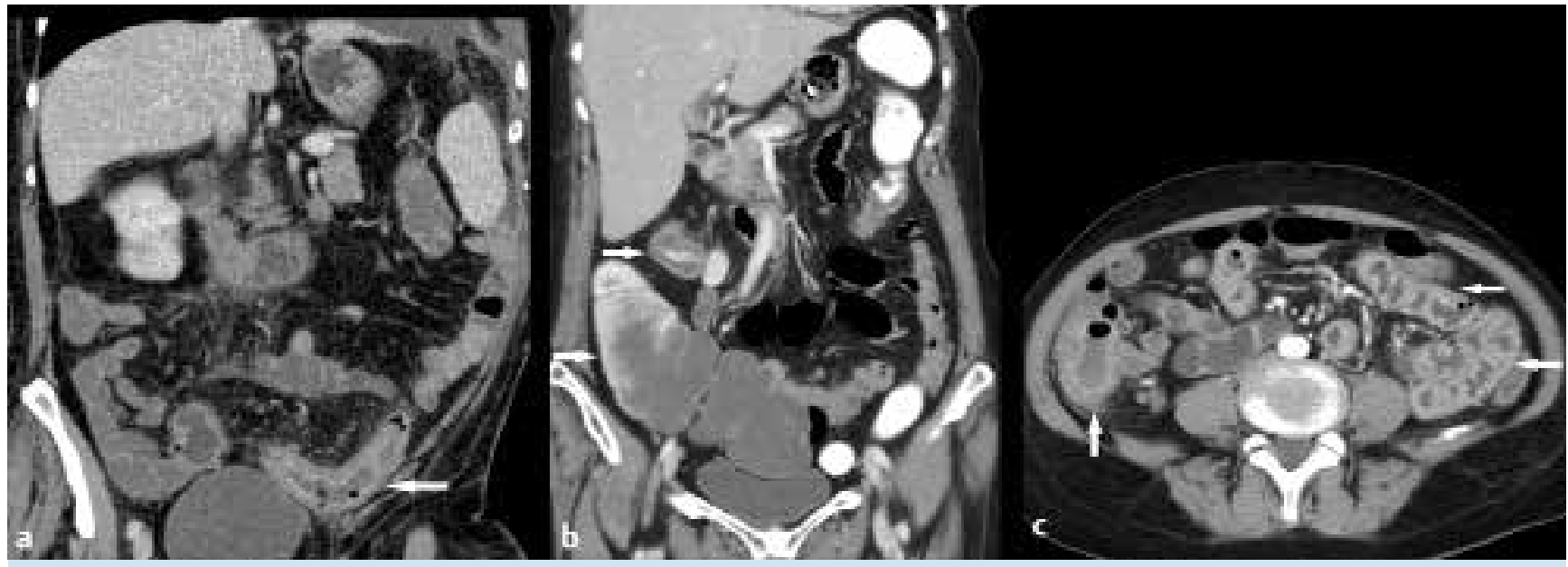

Abb. 8 Bildbeispiele von fokalen GI-Trakt-Infektionen durch Pilze (Mucormycose) (Pfeile).

\section{Infektiöse Komplikationen $\nabla$}

Pseudomembranöse Kolitis

Unter Chemotherapie und in der Aplasiephase sind bakterielle Pathogene die prädominanten Infektauslöser. Eine Antibiotikatherapie während dieser Phase kann zu einer Störung des Gleichgewichts der Darmflora mit Überwucherung durch Clostridium difficile führen. Die abgesonderten Clostridium-difficile-Toxine A und $B$ lösen eine Entzündungsreaktion aus, bei der es zur Exsudation von Fibrin und zur Ablagerung von Fibrinbelägen auf der Kolonschleimhaut kommt, was endoskopisch als Pseudomembranen imponiert. Klinisch manifestiert sich die Erkrankung mit wässrigen Diarrhoen, abdominellen Schmerzen und Fieber. Der häufigste computertomografische Befund ist eine deutliche Darmwandverdickung, die meistens das gesamte Kolon und weniger häufig nur das linke Hemikolon betrifft. In der frühen Phase kann ein segmentaler Befall nachgewiesen werden. Selten liegt auch eine Dünndarmbeteiligung vor. Dabei geht die Darmwandverdickung bei der pseudomembranösen Kolitis über solche bei anderen Kolitiden hinaus. Das sogenannte „accordion sign“, welches durch die Kontrastmittelanreicherung zwischen den Pseudomembranen und den geschwollenen Haustren zustande kommt, ist hoch-suggestiv auf eine pseudomembranöse Kolitis ( 0 Abb. 6 a). Da die Erkrankung vorwiegend die Mukosa und Submukosa betrifft, ist die parakolische Fettgewebsimbibierung dysproportional geringer ausgeprägt als die ausgeprägte Darmwandverdickung [Schmit $\mathrm{M}$ et al. AJR Am J Roentgenol 2008; 190: 712719; Donnelly LF. AJR Am J Roentgenol
1996; 167: 909-913; Coy DL et al. RadioGraphics 2005; 25: 305-318]. Bakterielle GI-Infektionen finden sich gehäuft auch in anderen Abschnitten des Verdauungstrakts ( $\odot$ Abb. $\mathbf{6 b}-\mathbf{e}$ ).

\section{Virale Infektionen}

Virale Pathogene sind eine häufige Ursache für Darminfektionen nach allogener Stammzelltransplantation, können aber auch in der Aplasiephase nach Chemotherapie auftreten. Die frequentesten Erreger sind das Cytomegalievirus (CMV), Adenovirus (ADV), Herpes-Simples-Virus (HSV), Rotavirus und Astrovirus. In der frühen Posttransplantationsphase (bis Tag 100 nach Transplantation) ist die CMV-Infektion die häufigste Ursache für Infekt bedingte gastrointestinale Komplikationen wie Diarrhoe, Hämorrhagie, Perforation und Peritonitis ( $\bullet$ Abb. 7). Die CMV-Kolitis manifestiert sich typischerweise als eine Darmwandverdickung im ileozökalen Übergang und im Colon ascendens. Häufig finden sich auch Schleimhautulzerationen. Der CT-Befund einer gastrointestinalen CMV-Infektion ähnelt dem einer Typhlitis mit einem ausgeprägten Zielscheibenphänomen, wobei angrenzender Aszites und Fettgewebsimbibierungen bei der gastrointestinalen CMV-Infektion deutlicher sind als bei der Typhlitis. Zudem kann begleitend eine Dünndarmwandverdickung vorhanden sein. Vergleichbare CT-Befunde sind auch bei der gastrointestinalen HSV-Infektion (10.-30. Tag nach Transplantation) zu finden. Hier ist die Dünndarmbeteiligung üblicherweise segmental mit angrenzender Fettgewebsimbibierung. Eine Rotavirus-Infektion präsen- tiert sich als eine segmentale Enteritis mit starker KM-Affinität der Schleimhaut, mäßiger angrenzender Fettgewebsimbibierung und intraluminaler Flüssigkeitsansammlung. Die ADV-Enteritis führt häufig zu Darmwandhämorrhagien [Schmit $M$ et al. AJR Am J Roentgenol 2008; 190: 712-719; Donnelly LF. AJR Am J Roentgenol 1996; 167: 909-913; Coy DL et al. RadioGraphics 2005; 25: 305-318].

\section{Pilzinfektionen}

Pilzinfekte treten typischerweise bei Patienten mit schwerer und lang anhaltender Neutropenie auf. Candida, Aspergillus und Mucor sind die häufigsten nachgewiesenen Erreger. Candida und Aspergillus verursachen erosive Infekte des GI-Trakts

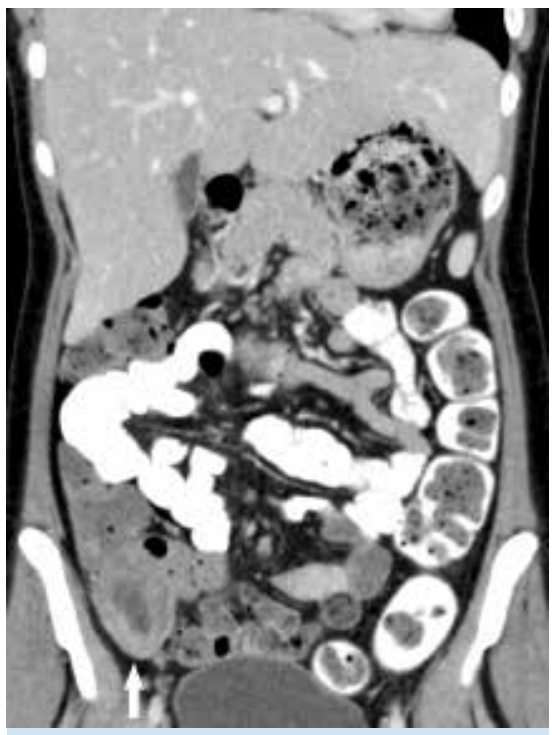

Abb. 9 Typhlitis in der Neutropenie mit zirkulärer Zökumwandverdickung (Pfeil). 
und betreffen bevorzugt den Oesophagus. Die Aspergillus-Kolitis neigt zur Infiltration von Blutgefäßen und verursacht Gefäßverschlüsse mit peripheren Hämorrhagiearealen und sekundären Infarktzonen [Schmit M et al. AJR Am J Roentgenol 2008; 190: 712-719]. Bei Patienten mit Neutropenie ist die Aspergillose nicht selten systemisch und kann bei Dissemination zu Mikroabszessen in Leber, Milz und Nieren führen. Invasive Candidiasis und Mucormycose verhalten sich pathogenetisch ähnlich ( $\triangle$ Abb. 8) [Coy DL et al. RadioGraphics 2005; 25: 305-318]. Die Mucormycose hat eine insgesamt niedrigere, jedoch aufgrund der antifungalen Prophylaxe und der hieraus resultierenden Selektion eine steigende Inzidenz. Bei der gastrointestinalen Mucormycose sind der Oesophagus und der Magen die am häufigsten betroffenen Organe. Das terminale Ileum und das Zökum sind bevorzugte Manifestationsorte einer intestinalen Mucormycose. Da die Erkrankung oft einen fulminanten und fatalen Verlauf hat, ist eine zügige Diagnostik und aggressive Therapie notwendig [Schmit $M$ et al. AJR Am J Roentgenol 2008; 190: 712-719]. Die CTDiagnostik zeigt eine zirkuläre Darmwandverdickung. Aufgrund der Koinzidenz von Nekrosen und Infarkten sowie Ödem und Inflammation, sind in der Darmwand gleichzeitig Zonen erhöhter und erniedrigter KM-Affinität zu finden.

\section{Typhlitis}

Typhlitis oder auch neutropene Kolitis ist eine lebensbedrohliche nekrotisierende Enteropathie, welche bei krankheitsassozierter oder therapieinduzierter Neutropenie beobachtet wird. Betroffen sind das Zökum und das Colon ascendens, eine Beteiligung des terminalen Ileums ist möglich. Eine Beteiligung weiterer Darmabschnitte ist unüblich. Pathophysiologisch liegt der Erkrankung eine Schwächung der Mukosa mit sekundärer bakterieller Invasion der Darmwand und evtl. späterer Bakteriämie bzw. Sepsis zugrunde. Schwere Fälle können zu Darmischämie und Perforation führen. Typische klinische Symptome stellen Fieber, blutige oder wässrige Durchfälle und rechtsseitige Unterbauchschmerzen dar [Schmit M et al. AJR Am J Roentgenol 2008; 190: 712-719; Hunter TB et al. AJR Am J

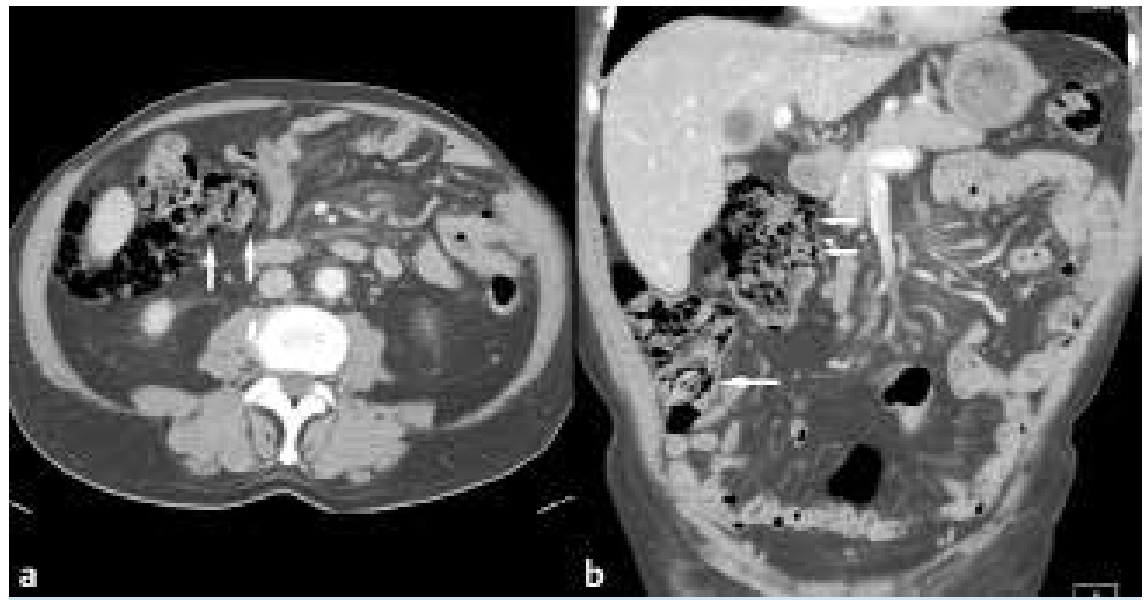

Abb. 10 Pneumatosis intestinalis bei 2 Patienten während der Chemotherapie. Beide Fälle wurden erfolgreich konservativ behandelt. Die intramurale Luft (Pfeile) war zum Teil auch nach retroperitoneal gelangt.

Roentgenol 1984; 142: 513-518; Kaste SC et al. AJR Am J Roentgenol 1999; 173: 729-736; Frick MP et al. AJR Am J Roentgenol 1984; 143: 763-765; Donnelly LF. AJR Am J Roentgenol 1996; 167: 909913; Coy DL et al. RadioGraphics 2005; 25: 305-318; Levine DS et al. RadioGraphics 2007; 27: 307-324].

Computertomografisch fällen Wandverdickungen der prädisponierten Darmabschnitte mit angrenzender entzündlicher Imbibierung des Fettgewebes sowie geringer parakolischer Aszites auf ( $\bullet$ Abb. 9). Eine lokoregionale Lymphadenopathie wird üblicherweise nicht beobachtet [Schmit M et al. AJR Am J Roentgenol 2008; 190: 712-719; Frick MP et al. AJR Am J Roentgenol 1984; 143: 763-765; Coy DL et al. RadioGraphics 2005; 25: 305-318].

\section{Pneumatosis intestinalis}

Pneumatosis intestinalis ist definiert als Gasansammlung in der Darmwand ( $\triangle$ Abb. 10). Es handelt sich nicht um eine Erkrankung, sondern um einen radiologischen und pathologischen Befund, welchem eine Vielzahl an Erkrankungen zugrunde liegen kann. Neben der Darmischämie und infektiösen Gastroenteritiden bzw. Kolitiden können Typhlitiden und akute Graft-versus-Host-Reaktionen diesen Befund verursachen. Außerdem können die bei Chemo- und Steroidtherapie- regimen auftretende Atrophie der PeyerPlaques und die daraus resultierenden Schleimhautdefekte das Eintreten intraluminaler Luft in die Darmwand begünstigen und so eine Pneumatosis intestinalis bedingen [Schmit M et al. AJR Am J Roentgenol 2008; 190: 712-719; Wood BJ et al. AJR Am J Roentgenol 1995; 165: 1387-1390; Keats TE et al. AJR Am J Roentgenol 1974; 122: 150-152; Day DL et al. AJR Am J Roentgenol 1988; 151: 8587; Bates FT et al. AJR Am J Roentgenol 1989; 152: 991-994]. Die Signifikanz der Pneumatosis intestinalis ist bei stammzelltransplantierten Patienten, insbesondere in der Neutropenie, eine andere mit deutlich günstigeren Verläufen trotz konservativer Therapie [Day DL et al. AJR Am J Roentgenol 1988; 151: 85-87; Bates FT et al. AJR Am J Roentgenol 1989; 152: 991-994].

\section{Zusamenfassung \\ $\nabla$}

Kenntnisse über die Art und Häufigkeit von GI-Komplikationen bei hämatologischen Patienten sowie eine enge interdisziplinäre Zusammenarbeit sind insbesondere in spezialisierten Zentren Voraussetzung für ein gelungenes Patienten-Management. In diesem Kontext spielt die Bildgebung oft eine entscheidende Rolle, da sie im Gegensatz zu anderen Diagnoseverfahren, z. B. Laboruntersuchungen, sofort verfügbar ist.

\section{Kurucay, C. Kloth, W. Bethge, C. Faul, M. Horger; Tübingen}

